\title{
Carcinoma ductal in situ de la mama. Análisis del período 1998-2002 en el Hospital Clínico Regional de Valdivia
}

\author{
Juan Antonio Pérez P, Sebastián Soto G.
}

\begin{abstract}
RESUMEN
El carcinoma ductal in situ de la mama se define como la proliferación de células neoplásicas en el interior de los ductos mamarios sin sobrepasar la membrana basal. Durante la última década, a nivel mundial, hemos presenciado un aumento progresivo de su incidencia, dado principalmente por el advenimiento del screening mamográfico. Se presentan las características clínicas, histopatológicas y la conducta terapéutica adoptada en 14 pacientes de sexo femenino, portadoras de esta patología entre los años 1998 y 2002, en el Hospital Clínico Regional de Valdivia. La edad promedio de las mujeres fue de 39 años con un margen entre 25 y 88 años. Diez pacientes presentaron masa palpable, siendo 7 casos detectados por autopalpación y 3 por examen físico mamario. La localización más frecuente correspondió a la mama izquierda, en 8 casos. En 12 pacientes se realizó mamografía, siendo catalogada como BI-RADS IV en 10 de ellas. En 7 casos se realizó citología por punción aspirativa, resultando todas sospechosas de cáncer. Las 14 pacientes fueron sometidas a cirugía, practicando en 8 de ellas una mastectomía simple extendida. Se presentó una complicación menor y en la serie no hubo mortalidad. El diagnóstico definitivo de carcinoma ductal in situ se certificó con el estudio histopatológico diferido de la pieza operatoria, siendo el tipo comedo el más frecuente. Once pacientes recibieron quimioprofilaxis con tamoxifeno $20 \mathrm{mg} / \mathrm{dí}$. Actualmente ninguna de las pacientes presenta evidencias de enfermedad. (Palabras claves/Key words: Carcinoma ductal in situ/Ductal in situ carcinoma; Cáncer de mama/Breast cancer; Tamoxifeno/Tamoxifen).
\end{abstract}

\section{INTRODUCCIÓN}

El carcinoma ductal in situ de la mama (CDIS), también conocido como carcinoma intraductal, se define como la proliferación de células neoplásicas confinada al interior de los ductos mamarios, respetando la membrana basal'-3.

Por décadas esta patología fue subdiagnosticada, exceptuando los casos que se manifestaban como masa palpable y/o descarga por pezón, situación que hoy en día ocurre en menos del $20 \%$ de los CDIS diagnosticados anualmente a nivel mundial ${ }^{1}$. Durante las décadas de los '70 y ' 80 el CDIS no superaba el $10 \%$ del total de cánceres de mama registrados anualmente en Estados Unidos de Norteamérica², sin embargo, en la actualidad, con la masificación del screening mamográfico, esta neoplasia representa entre el 25 a $30 \%$ de todos los carcinomas mamarios, no existiendo diferencias significativas en cuanto a la edad de presentación con respecto al carcinoma mamario corriente ${ }^{2}$.

EI CDIS habitualmente se comporta como neoplasia benigna desde el punto de vista de la mortalidad ya que ésta no supera el $1 \%$. Tal situación obedece a que al estar confinadas las células neoplásicas al interior de los ductos mamarios no se encuentran en contacto con vasos 
linfáticos, por lo cual las posibilidades de metastizar son nulas, siendo ésta la base fisiopatológica para considerar que la biopsia del linfonodo centinela en este tipo de carcinoma es innecesaria ${ }^{3}$. Cabe destacar que aquellos CDIS que recidivan post cirugía o que presentan metástasis al momento del diagnóstico, se explican como consecuencia de la multicentricidad en el primer caso y a raíz de focos de carcinoma invasor no detectados en el estudio histopatológico en el segundo $0^{1-3}$.

El riesgo que tiene el CDIS de progresar a cáncer invasor, depende de una combinación de factores, como el tipo histológico, tamaño de la lesión y estado del margen de resección.

A pesar de los grandes avances tecnológicos desarrollados en la medicina contemporánea tendientes a detectar precozmente este tipo de cáncer, la mamografía continúa siendo el examen de elección para el diagnóstico del CDIS, puesto que posibilita el hallazgo de microcalcificaciones de patrón granular o lineal ${ }^{4}$ que proporcionan la alerta para sospechar la enfermedad. Otras condiciones benignas o premalignas de la mama pueden estar asociadas a un patrón similar de calcificaciones incluyendo la adenosis esclerosante y la hiperplasia ductal atípica. Cuando existen imágenes mamográficas sospechosas no palpables, los procedimientos más corrientes para su diagnóstico son la radiología intervencionista (biopsia estereotáxica) y la biopsia radioquirúrgica.

El tratamiento del CDIS permanece controversial. Se acepta que la base de la terapia es la cirugía. Las técnicas realizadas incluyen cirugía conservadora exclusiva, cirugía conservadora asociada a radioterapia y la mastectomía total, fundamentándose la elección del tipo de operación principalmente en la magnitud de la neoplasia. La quimioprofilaxis con tamoxifeno resultaría fundamental hoy en día en pacientes con receptores estrogénicos positivos, ya que ha demostrado que reduce el riesgo de recurrencia hasta en un $40 \% 5$.

El objetivo de este trabajo es mostrar las características clínicas y patológicas de las pacientes portadoras de CDIS manejadas en el Hospital Clínico Regional de Valdivia en un período de 5 años.

\section{MATERIAL Y MÉTODOS}

Revisión retrospectiva de los registros clínicos y patológicos de las pacientes portadoras de CDIS diagnosticados en el período comprendido entre Enero 1998 y Diciembre 2002.
Los datos fueron ingresados a una planilla Microsoft Excel y se analizaron los siguientes parámetros: edad, sexo, antecedentes mórbidos personales y familiares, mecanismo de detección, tiempo entre el diagnóstico y la cirugía, localización, exámenes realizados previo a la cirugía, tipo de cirugía y terapia adyuvante.

\section{RESULTADOS}

En el período que comprendió el estudio se registraron 14 pacientes de sexo femenino con edad promedio de 39 años (margen 25 a 88 años). Dos pacientes relataron antecedentes de patología neoplásica familiar, una de cáncer de mama materno y otra de cáncer pulmonar paterno, correspondiendo cada una al $7,1 \%$.

En 10 pacientes $(71,4 \%)$ el CDIS se manifestó como tumor mamario, cuyo diámetro mayor promedio fue de $4,6 \mathrm{~cm}$, con margen entre 1 y $12 \mathrm{~cm}$. En 7 casos la detección del tumor fue mediante autopalpación y en 3 por examen físico de mamas. Sólo 4 casos se pesquisaron a través del screening mamográfico que detectó microcalcificaciones sospechosas.

La localización más frecuente fue la mama izquierda en 8 casos $(57,1 \%)$.

En 12 pacientes se realizó mamografía (Figura 1), siendo clasificada en 10 casos como BI-RADS IV $(71,4 \%)$, y en 2 como BI-RADS V $(14,3 \%)$.

En 7 pacientes (50\%) se realizó citología por punción aspirativa con aguja fina, la cual en todos los casos fue positiva, contribuyendo a ratificar la sospecha clínica de patología neoplásica maligna.

El diagnóstico definitivo se confirmó en todos los casos a través del estudio histopatológico diferido de la pieza operatoria (Figura 2), siendo el subtipo comedo el más frecuente con 9 casos $(64,3 \%)$.

Solamente se presentó una complicación relacionada con la cirugía que correspondió a una distensión del plexo braquial, la cual evolucionó favorablemente quedando la enferma sin secuelas.

En 11 pacientes $(78,6 \%)$ los receptores estrogénicos fueron positivos, quedando todas ellas con quimioprofilaxis con tamoxifeno $20 \mathrm{mg} / \mathrm{día}$.

En la Tabla 1 se describen las características de las 14 pacientes con CDIS.

Actualmente las 14 pacientes de la serie se encuentran en buenas condiciones, estando todas sin evidencias de enfermedad. 


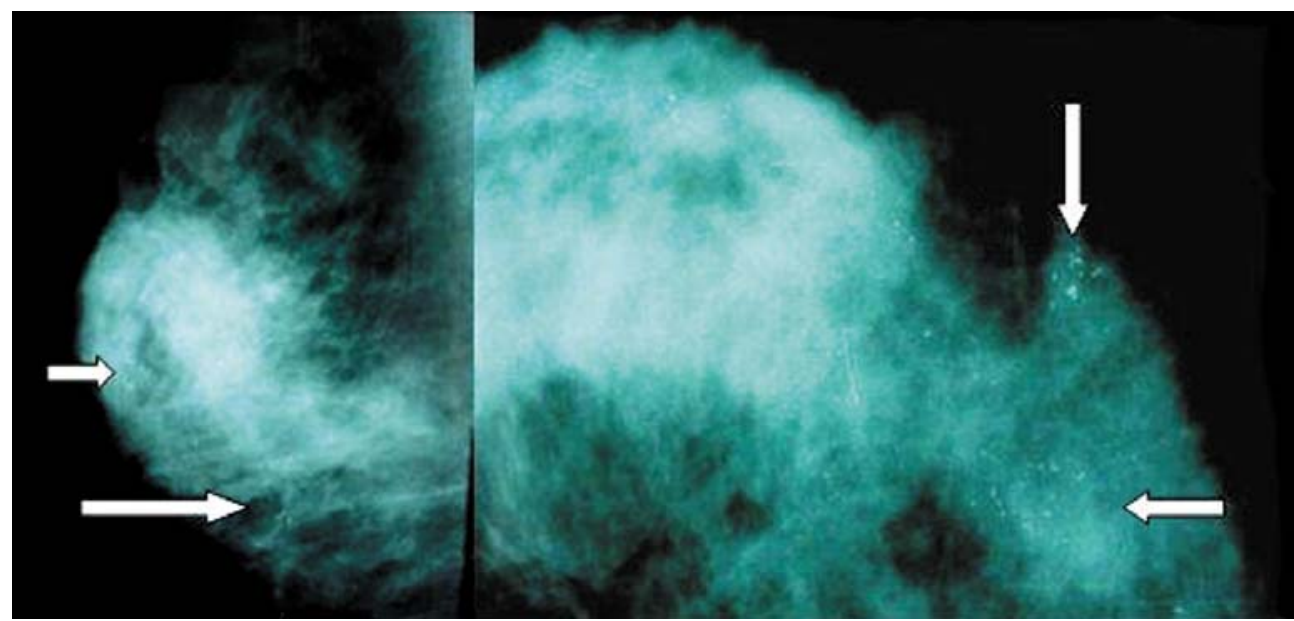

FIGURA 1. Mamografía en proyecciones centrolateral y craneocaudal con microcalcificaciones sospechosas de CDIS (flechas).

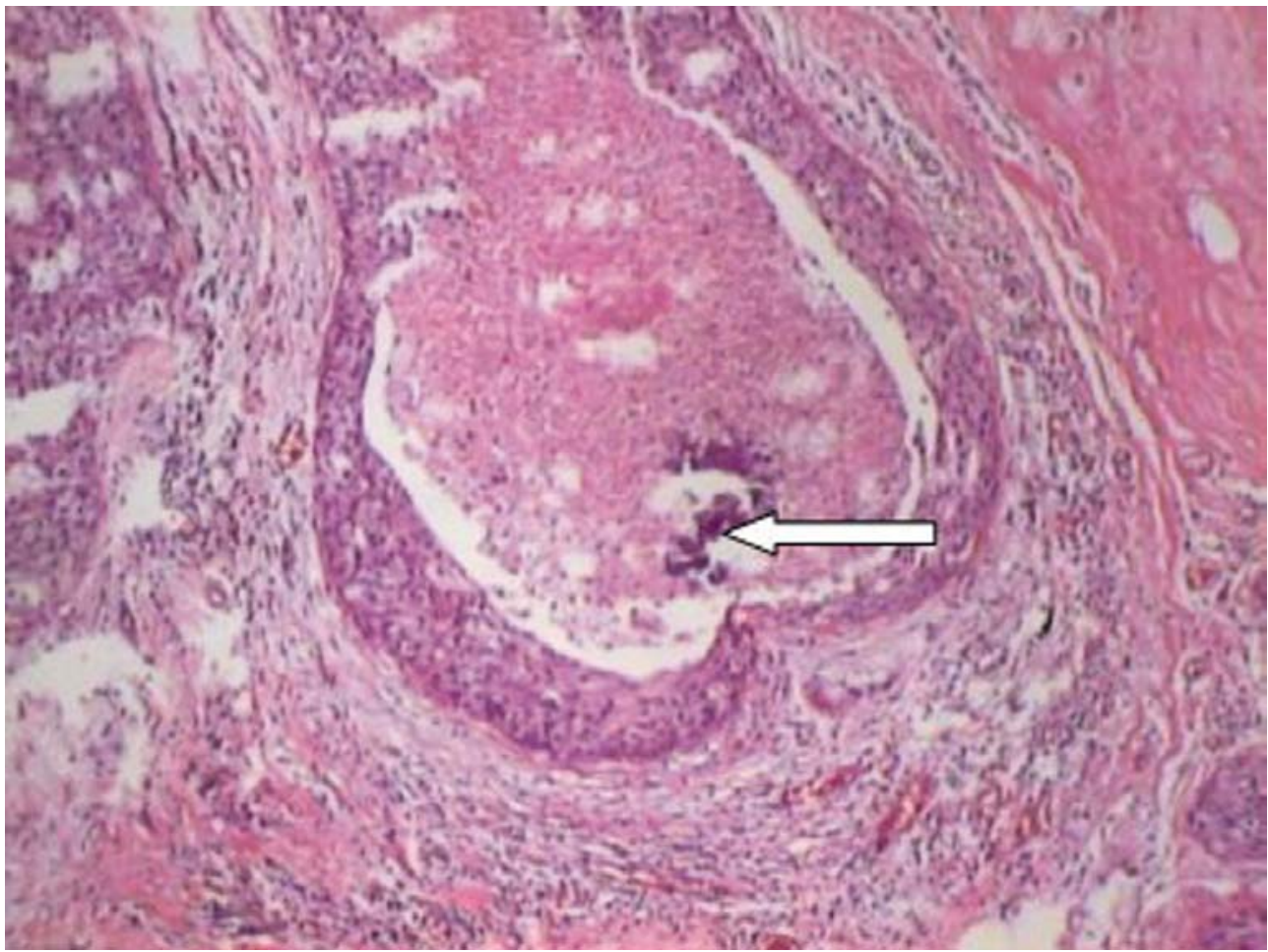

FIGURA 2. Corte histológico de espécimen operatorio, en que se identifica ducto mamario seccionado transversalmente con focos de CDIS tipo comedo. La flecha indica foco de necrosis y calcificación al interior de la proliferación celular neoplásica. 
Tabla 1. Características clínicas y patológicas de 14 pacientes portadoras de CDIS.

\begin{tabular}{|c|c|c|c|c|c|c|c|c|c|c|}
\hline Caso & Edad & Antecedentes & Detección & Mamografía & Diagnóstico & Cirugía & Complicación & Tipo & $\mathrm{RE}^{*}$ & TMF $^{\star *}$ \\
\hline 1 & 33 & $\begin{array}{l}\text { Cáncer mama } \\
\text { (Madre) }\end{array}$ & Mamografía & BI-RADS IV & $\begin{array}{l}\text { Biopsia } \\
\text { radioquirúrgica }\end{array}$ & $\begin{array}{l}\text { Mastectomía total } \\
\text { + prótesis }\end{array}$ & No & $\begin{array}{l}\text { Comedo, } \\
\text { alto grado }\end{array}$ & + & Sí \\
\hline 2 & 48 & - & Autopalpación & BI-RADS IV & $\begin{array}{l}\text { Citología por } \\
\text { punción }\end{array}$ & $\begin{array}{l}\text { Mastectomía total } \\
\text { + prótesis }\end{array}$ & No & $\begin{array}{l}\text { Comedo, } \\
\text { grado } \\
\text { intermedio }\end{array}$ & + & Sí \\
\hline 3 & 50 & - & Mamografía & BI-RADS IV & $\begin{array}{l}\text { Biopsia } \\
\text { radioquirúrgica }\end{array}$ & $\begin{array}{l}\text { Mastectomía } \\
\text { parcial }\end{array}$ & No & Cribiforme & + & Sí \\
\hline 4 & 37 & $\begin{array}{l}\text { Mastopatía } \\
\text { fibroquística } \\
\text { (Madre) }\end{array}$ & Mamografía & BI-RADS IV & $\begin{array}{l}\text { Biopsia } \\
\text { radioquirúrgica }\end{array}$ & $\begin{array}{l}\text { Mastectomía } \\
\text { simple extendida } \\
\text { + prótesis }\end{array}$ & $\begin{array}{l}\text { Distensión } \\
\text { plexo braquial }\end{array}$ & Comedo & + & Sí \\
\hline 5 & 37 & - & $\begin{array}{l}\text { Examen físico } \\
\text { mamas }\end{array}$ & BI-RADS V & $\begin{array}{l}\text { Citología por } \\
\text { punción }\end{array}$ & $\begin{array}{l}\text { Mastectomía } \\
\text { simple extendida }\end{array}$ & No & Cribiforme & + & Sí \\
\hline 6 & 50 & - & Autopalpación & BI-RADS IV & $\begin{array}{l}\text { Citología por } \\
\text { punción }\end{array}$ & $\begin{array}{l}\text { Mastectomía } \\
\text { simple extendida }\end{array}$ & No & Micropapilar & - & No \\
\hline 7 & 67 & - & Autopalpación & BI-RADS IV & - & $\begin{array}{l}\text { Mastectomía } \\
\text { simple extendida }\end{array}$ & No & $\begin{array}{l}\text { Comedo, } \\
\text { alto grado }\end{array}$ & + & Sí \\
\hline 8 & 88 & - & $\begin{array}{l}\text { Examen físico } \\
\text { mamas }\end{array}$ & BI-RADS IV & $\begin{array}{l}\text { Citología por } \\
\text { punción }\end{array}$ & $\begin{array}{l}\text { Mastectomía } \\
\text { parcial }\end{array}$ & No & Micropapilar & + & Sí \\
\hline 9 & 77 & - & $\begin{array}{l}\text { Examen físico } \\
\text { mamas }\end{array}$ & BI-RADS V & - & $\begin{array}{l}\text { Mastectomía } \\
\text { simple extendida }\end{array}$ & No & $\begin{array}{l}\text { Comedo, } \\
\text { grado } \\
\text { intermedio }\end{array}$ & - & No \\
\hline 10 & 34 & - & Mamografía & BI-RADS IV & $\begin{array}{l}\text { Biopsia } \\
\text { radioquirúrgica }\end{array}$ & $\begin{array}{l}\text { Mastectomía } \\
\text { simple extendida }\end{array}$ & No & Comedo & - & No \\
\hline 11 & 53 & TBC (Hermana) & Autopalpación & BI-RADS IV & $\begin{array}{l}\text { Citología por } \\
\text { punción }\end{array}$ & $\begin{array}{l}\text { Mastectomía } \\
\text { simple extendida }\end{array}$ & No & $\begin{array}{l}\text { Comedo, } \\
\text { alto grado }\end{array}$ & - & No \\
\hline 12 & 48 & - & Autopalpación & BI-RADS IV & $\begin{array}{l}\text { Citología por } \\
\text { punción }\end{array}$ & $\begin{array}{l}\text { Mastectomía } \\
\text { simple extendida }\end{array}$ & No & Cribiforme & + & Sí \\
\hline 13 & 27 & - & Autopalpación & - & $\begin{array}{l}\text { Citología por } \\
\text { punción }\end{array}$ & $\begin{array}{l}\text { Mastectomía } \\
\text { simple extendida }\end{array}$ & No & $\begin{array}{l}\text { Comedo, } \\
\text { alto grado }\end{array}$ & + & Sí \\
\hline 14 & 25 & . & Autopalpación & . & $\begin{array}{l}\text { Citología por } \\
\text { punción }\end{array}$ & $\begin{array}{l}\text { Mastectomía } \\
\text { simple extendida }\end{array}$ & No & $\begin{array}{l}\text { Comedo, } \\
\text { grado } \\
\text { intermedio }\end{array}$ & - & No \\
\hline
\end{tabular}

*RE: receptor estrógeno $\quad{ }^{* *}$ TMF: tamoxifeno

\section{DISCUSIÓN}

A partir de las primeras descripciones patológicas del CDIS realizadas por el cirujano inglés J.C. Warren, a principios del siglo XX, son múltiples los estudios que se han llevado a cabo con el fin de determinar el comportamiento biológico y la terapia más efectiva para esta patología $a^{2,6}$.

En el estudio histopatológico se diferencian 5 subtipos cuyas características se describen en la Tabla 2.
De significativa importancia se ha considerado la clasificación nuclear, que se muestra en la Tabla 3, puesto que orienta la decisión terapéutica y refleja el potencial de recurrencia post tratamiento ${ }^{7}$.

Los factores pronósticos que se han mencionado como relevantes en el $\mathrm{CDIS}^{3-5}$ se resumen en la Tabla 4.

Los reportes sobre la incidencia de multicentricidad del CDIS en especímenes de mastectomía llegan hasta el $47 \%$. En las lesiones detectadas mamográficamente, la 
Tabla 2. Clasificación histológica del CDIS ${ }^{1,2}$.

\begin{tabular}{|ll|}
\hline Tipos & Características \\
\hline Micropapilar & Células neoplásicas se agrupan y protruyen hacia el lumen del ducto. \\
Cribiforme & Proliferación de células neoplásicas formando microlúmenes al interior del ducto. \\
Comedo & Crecimiento sólido, pobremente diferenciado con focos de necrosis y calcificación. \\
Sólido & Células neoplásicas ocupan completamente el lumen con o sin necrosis y \\
Papilar & calcificación. \\
\hline
\end{tabular}

Tabla 3. Clasificación nuclear ${ }^{1,2}$.

\begin{tabular}{|llll|}
\hline Características nucleares & Alto Grado & Grado Intermedio & Bajo Grado \\
\hline Diámetro nuclear (micrones) & $>2,5-3$ & $2-2,5$ & $<2$ \\
Pleomorfismo & Prominente & Uniforme & Ausente \\
Cromatina & Vesicular & Tosco & Difuso \\
Nucléolo & Prominente & Pequeño & Ausente \\
Mitosis & Frecuente & Infrecuente & Raro \\
\hline
\end{tabular}

Tabla 4. Factores pronósticos ${ }^{3-5}$.

Multicentricidad: $21-32 \%$ de los casos.

Invasión oculta: $21-32 \%$ de los casos.

Tipo comedo. Invasión oculta en $25 \%$ de los casos.

Grado nuclear. A mayor grado peor es el pronóstico.

Tamaño tumoral. Prácticamente el $100 \%$ de los tumores mayores de $2,5 \mathrm{~cm}$ son multicéntricos.

frecuencia de multicentricidad parece estar relacionada con el tamaño de la lesión. En una serie se encontró que la multicentricidad fue comparativamente más frecuente en los CDIS mayores de $2,5 \mathrm{~cm}$, que en los de tamaño menor (54 versus 14 por ciento), siendo la media del tamaño de las lesiones en los 2 grupos de 5,6 y $0,8 \mathrm{~cm}$ respectivamente 6 .

Como resultado de múltiples estudios ya realizados o en curso, se ha logrado determinar ciertas características clínicas y patológicas que permiten optimizar la terapia para cada paciente. La cirugía conservadora con o sin radioterapia asociada a quimioprofilaxis con tamoxifeno 20 $\mathrm{mg} /$ día por 5 años, sería la terapia de elección siempre que los casos que sean tributarios de ella ${ }^{1,7-15}$. La principal contraindicación para este tipo de cirugía son los CDIS extensos, puesto que los resultados cosméticos son inaceptables.

Indicaciones de tratamiento conservador ${ }^{1}$ :

1. Área pequeña de microcalcificaciones medidas en el examen histopatológico o en la mamografía, preferentemente no mayor a 2 a $3 \mathrm{~cm}$.
2. Patrón irregular de microcalcificaciones con área menor de $6 \mathrm{~cm}^{2}$.

3. Que se pueda obtener con la cirugía un margen libre de CDIS de al menos $10 \mathrm{~mm}$.

4. Grado nuclear bajo o intermedio.

5. Mama estéticamente aceptable post cirugía.

Los casos de recurrencia, luego de tratamiento conservador, se deben generalmente a márgenes de sección inadecuados 0 microinvasión oculta. Se define como microinvasión los focos de cáncer invasor con diámetros de 1 milímetro o menos y corresponde al T1 mic dentro del sistema TNM. Es conocido que el $1 \%$ de las pacientes sometidas a esta terapia tienen riesgo de desarrollar cáncer invasor, lo que no significa que CDIS sea precursor de éste como se creía en los primeros años de estudio de esta patología ${ }^{1}$.

La mastectomía total tiene indicaciones específicas en esta patología y en la actualidad se realiza en alrededor del $25 \%$ de las pacientes con CDIS'.

Las indicaciones para realizar este tipo de cirugía son: 
1. CDIS extenso, en el cual es imposible lograr márgenes oncológicos sin alterar la estética de la mama.

2. CDIS multicéntrico.

3. Contraindicación de radioterapia (enfermedad del colágeno, radioterapia previa).

La mastectomía total (simple) se asocia con un porcentaje de la cura de 98 a $99 \%$ para todos los tipos de DCIS.

Es importante destacar que el tratamiento de la axila en esta patología es innecesario, ya que al no estar las células neoplásicas en contacto con vasos linfáticos no dan metástasis nodales $^{1-2}$.

Como el CDIS representa un grupo heterogéneo de lesiones de potencial maligno variable en el momento de tomar la decisión terapéutica, es prudente tener en cuenta ciertas consideraciones:

- Pacientes con DCIS localizado son candidatos para cirugía conservadora e irradiación. La interpretación detallada de la mamografía y la evaluación cuidadosa patológica son esenciales para confirmar la naturaleza localizada de la lesión y juzgar la suficiencia de resección. Las metas de la cirugía son remover todas las microcalcificaciones sospechosas y lograr márgenes negativos en la resección.

La escisión exclusiva puede ser un tratamiento apropiado para mujeres seleccionadas que presentan lesiones pequeñas de DCIS (menos de 1 a $2 \mathrm{~cm}$ ), de bajo grado, resecada con márgenes claramente negativos. Los datos del protocolo B-17 del NSABP sugieren que incluso estas pacientes se benefician con la radioterapia y que la determinación para proceder a la radiación debe fundamentarse en consideraciones clínicas y en la decisión de las pacientes, una vez que han sido debidamente informadas ${ }^{16}$.

- En las mujeres con lesiones de alto grado tiene indicación la mastectomía total, con un muestreo nodal axilar bajo lo cual obvia la necesidad de una reoperación si se identifica que existe invasión. La disección axilar no está indicada.

- Debe considerarse el uso de tamoxifeno para reducir el riesgo de tumor recurrente ipsilateral después de la cirugía conservadora y para reducir el riesgo de cáncer de mama contralateral en todos los pacientes.
Respecto a nuestra serie, si consideramos que el DCIS puede presentarse como masa palpable, descarga patológica de pezón con o sin una masa, descubrimiento incidental en una biopsia de mama realizada para diagnosticar otra patología, o hallazgo de una lesión subclínica en una mamografía de rutina, lo que corresponde actualmente a la mayoría de los casos diagnosticados en los centros en que se utiliza este recurso, llama la atención que en nuestra casuística el carcinoma se diagnosticó en la mayoría de los casos cuando se manifestó en forma clínica, lo que por una parte traduce un deficiente screening mamográfico y explica la extensión de la cirugía que fue realizada.

En la mitad de nuestras pacientes se obtuvo citología por punción aspirativa, resultando todas compatibles con cáncer de mama. A pesar de que con este procedimiento es imposible diferenciar entre CDIS y cáncer invasor, lo que implica una terapéutica diferente, no tiene gran implicancia debido a lo tardío de la primera consulta.

Sólo 2 enfermas fueron tributarias de tratamiento conservador, debido a que la gran mayoría de ellas consultaron por masa palpable voluminosa ( $4,6 \mathrm{~cm}$ en promedio), la que orienta en un primer momento hacia un cáncer mamario invasor, por lo cual el enfrentamiento apuntó en esa dirección y no al CDIS.

Siendo la mastectomía simple extendida la cirugía más utilizada en nuestra serie, el procedimiento conlleva extirpar linfoganglios, lo cual no es necesario en esta patología como ya se mencionó anteriormente. En 10 casos se obtuvieron entre 4 y 15 linfoganglios con un promedio de 10 , resultando todos negativos para metástasis, lo que reafirma uno de los pilares que caracterizan esta patología, que es el hecho el CDIS no da metástasis nodales por las causas previamente mencionadas ${ }^{1-2}$.

La reconstrucción mamaria inmediata que actualmente se preconiza en el manejo de este tipo de pacientes ${ }^{1,3,8}$, sólo fue realizada en 3 pacientes, lo cual nos enfrenta a otro desafío que tenemos por delante.

De las 14 pacientes de nuestra serie, 11 tuvieron receptores estrogénicos positivos, indicándose en todas ellas quimioprofilaxis con tamoxifeno por un plazo no inferior a 5 años, conducta que está avalada por múltiples estudios internacionales ${ }^{1,5,17}$.

El análisis de nuestros casos nos indica que aún no alcanzamos un nivel adecuado de 
diagnóstico de esta patología, ya que anualmente en promedio corresponde a un 7\% de todos los cánceres de mama operados en nuestro servicio, lo que está muy por debajo del 25 a $30 \%$ descrito internacionalmente ${ }^{2}$. Lo anterior se explica en parte por el aún deficiente acceso que tiene nuestra población al screening mamográfico, a pesar que existen mejorías en las políticas de salud actualmente vigentes.

El seguimiento de nuestras enfermas se realizó mediante mamografía y examen clínico cada 6 meses durante el primer año y luego anualmente de por vida.

Finalmente debemos tener presente que esta patología no sólo se presenta en la mujer, ya que en el hombre representa el $1 \%$ del total de cánceres de mama que los afectan. Generalmente se presenta en mayores de 65 años, con masa palpable y descarga por pezón, siendo el subtipo comedo menos frecuente que en la mujer y el tratamiento siempre es la mastectomía 1 .

\section{REFERENCIAS}

1. The Consensus Conference Committee: Consensus Conference on the Treatment of in situ Ductal Carcinoma of the Breast, April 22-25, 1999. Cancer 2000; 88: 946-54

2. Rosen's PP: Intraductal Carcinoma. Rosen's Breast Pathology. Philadelphia, Lippincott-Raven Publishers, 1997; 227-69

3. Lagios M, Silverstein M: Sentinel node biopsy for patients with DCIS: A dangerous and unwarranted direction. Ann Surg Oncol 2001; 8: 275-7

4. Harris E, Solin L: The diagnosis and treatment of ductal carcinoma in situ of the breast. Breast $J$ 2000; 6: 78-95

5. Wilcken $\mathrm{N}$ : Tamoxifen hits the target in situ. Lancet 1999; 353: 1986-7

6. Lagios MD, Westdahl PR, Margolin FR, Rose MR: Duct carcinoma in situ. Relationship of extent of noninvasive disease to the frequency of occult invasion, multicentricity, lymph node metastases, and short-term treatment failures. Cancer 1982; 50: 1309-14

7. Page D, Simpson J: Ductal carcinoma in situ. The focus for prevention, screening, and breast conservation in breast cancer. $N$ Engl $\mathrm{J}$ Med 1999; 340: 1499-500

8. The Consensus Conference on the Classification of Ductal Carcinoma in situ, April 25 - 28, 1997. Cancer 1997; 80: 1798-802

9. Morrow M: Understanding ductal carcinoma in situ: A step in the right direction. Cancer 1999; 86: 375-7

10. Silverstein M, Gierson E, Colburn W, Cope L, Furmanski M, Senofsky G et al: Can intraductal breast carcinoma be excised completely by local excision? Clinical and pathologic predictors. Cancer 1994; 73: 2985-9

11. Silverstein M, Lagios M, Groshen S, Waisman J, Lewinsky B, Martino $S$ et al: The Influence of margin width on local control of ductal carcinoma in situ of the breast. $N$ Engl J Med 1999; 340: 1455-61

12. Page D, Dupont W, Rogers L, Jensen R, Schuyler $\mathrm{P}$ : Continued local recurrence of carcinoma 15-25 years after a diagnosis of low grade ductal carcinoma in situ of the breast treated only by biopsy. Cancer 1995; 76: 1197- 200

13. Neuschatz A, Di Petrillo T, Steinhoff M, Safaii $H$, Yunes $M$, Landa $M$ : The value of breast lumpectomy margin assessment as a predictor of residual tumor burden in ductal carcinoma in situ of the breast. Cancer 2002; 94: 1917-24

14. Hetelekidis S, Collins L, Silver B, Manola J, Gelman R, Cooper A: Predictors of local recurrence following excision alone for ductal carcinoma in situ. Cancer 1999; 85: 427-31

15. Fisher B, Dignam J, Wolmark N, Wickerham L, Fisher E, Mamounas E: Tamoxifen in treatment of intraductal breast cancer: National Surgical Adjuvant Breast and Bowel Project B-24 randomizsed controlled trial. Lancet 1999; 353: 1993- 2000

16. Fisher B, Dignam J, Wolmark N, Mamounas $\mathrm{E}$, Costantino $\mathrm{J}$ et al: Lumpectomy and radiation therapy for the treatment of intraductal breast cancer: findings from National Surgical Adjuvant Breast and Bowel Project B-17. J Clin Oncol 1998;16: 441-52

17. Arun B, Hortobagyi G: Progress in breast cancer chemoprevention. Endocrine-Related Cancer 2002; 9: 15-32 